\title{
A Design Approach for a Valve Based on a Magnetic Shape Memory Actuator
}

\author{
Annabell Effner ${ }^{1} \cdot$ Tobias Zawada $^{2} \cdot$ Jürgen Weber $^{1}$
}

Published online: 3 March 2020

(C) The Author(s) 2020

\begin{abstract}
With the motivation to meet the increasing requirements on fast-switching pneumatic valves, Magnetic Shape Memory alloys (MSM) are considered as a promising alternative to conventional actuators. Regarding the desired strokes, forces and actuation frequencies MSM alloys meet the requirements best among other solid-state transducers for valve applications. However, the hysteresis in the magneto-mechanical behavior is challenging during the sizing process of a component based on an MSM actuator. It has proved to be valuable to apply a system simulation including a lumped element model of the MSM element in the early design phase. In this contribution, a design approach for the valve based on an MSM actuator is shown. A system network model was used to design a functional prototype of the valve to achieve the specifications defined within a requirements analysis. These specifications were derived from characteristic features of conventional established fast-switching pneumatic valve. The analysis of the subsystems was done to exploit the deviations and their influences on the system behavior. Furthermore, the experimental research of the functional
\end{abstract}

This article is an invited submission to Shape Memory and Superelasticity selected from presentations at the International Conference on Ferromagnetic Shape Memory Alloys (ICFSMA) held June 2-7, 2019 in Prague, Czech Republic, and has been expanded from the original presentation.

Annabell Effner

annabell.effner@tu-dresden.de

Tobias Zawada

Tobias.Zawada@esi-group.com

1 Chair of Fluid-Mechatronic Systems, TU Dresden, 01062 Dresden, Germany

2 ESI ITI GmbH, 01067 Dresden, Germany prototype of the valve was carried out to check for functionality and to evaluate whether the specifications were met. A nominal volume flow of $120 \mathrm{Nl} / \mathrm{min}$ was reached at a supply Voltage $U$ of $48 \mathrm{~V}$ and current $i$ of $7.6 \mathrm{~A}$ which exceeds the aim of $100 \mathrm{Nl} / \mathrm{min}$. By means of an optimization of the actuators geometry the response time could be reduced to less than $1 \mathrm{~ms}$. The desired maximum modular dimension of $9 \mathrm{~mm}$ was not achieved by the functional prototype due to the available production process, but would be feasible with series production.

Keywords Magnetic shape memory $\cdot$ Mechanical behavior $\cdot \mathrm{NiMaGa} \cdot$ Pneumatic valve $\cdot$ Fast switching . MSM actuator

\section{Introduction}

Due to the progress on the material research of MSM alloys within the last two decades [1], the interest for the use of actuators based on MSM alloys as an alternative to conventional actuators increases. The large magnetic field induced strain compared to other solid-state transducers, high-dynamic properties, durability and variability in operation mode make MSM-based actuators especially attractive for applications that are currently restricted by a lower performance of conventional actuators. Considerable potential applications and design concepts have already been published. For example, a miniature short-circuit breaker that combines the functionality of two actuators in one is presented in [2]. Another idea is to use a local deformation for a miniaturized peristaltic pump to achieve accurate and adjustable dosing [3]. Basic design concepts are discussed in [4]. However, the design process is still complex due to the magneto-mechanical hysteresis of the 
MSM alloy, which can be a major obstacle in the design process to exploit the benefits of these materials. Although different mathematical approaches to describe the magneto-mechanical hysteresis are known, e.g. [5]. and [6], a design tool for a system engineer is still absent. For a successful application of the MSM-based actuators a design tool is needed to handle enough data about the material and provides a flexible access to these data making them easy-editable. This paper presents a design approach for system sizing based on MSM actuators. We illustrate a procedure to overcome the difficulties associated with the MSM characteristic by means of the design of a pneumatic switching valve.

\section{Materials and Methods}

\section{MSM Material and Design Process}

The MSM elements used for this study are specimens cut from the NiMnGa-alloy, provided by ETO Magnetics. With typical sizes of $2 \times 3 \times 15 \mathrm{~mm}^{3}$, external flux densities below $1 \mathrm{~T}$, strains of $6 \%$ at compressive stresses $\sigma$ up to $2 \mathrm{MPa}$, strokes $s_{\mathrm{MSM}}$ of almost $1 \mathrm{~mm}$ and forces $F_{\mathrm{MSM}}$ of $10 \ldots 20 \mathrm{~N}$ at frequencies more than $1 \mathrm{kHz}$ can be attained by an MSM element, suiting the desired strokes, forces and dynamic properties of pneumatic valves.

The workflow of the design process is shown in Fig. 1.

As in any other engineering task, a requirements analysis at the beginning of the design process is essential. Respective to the state of the art of established fastswitching pneumatic valves, the following requirements were specified as targets: a minimal response time under $1 \mathrm{~ms}$, a minimal modular dimension under $9 \mathrm{~mm}$ and a nominal volume flow of at least $100 \mathrm{Nl} / \mathrm{min}$ at a maximum supply voltage of $48 \mathrm{~V}$. Moreover, a high duty cycle, and therefore, a thermal resistance of the MSM-based actuator

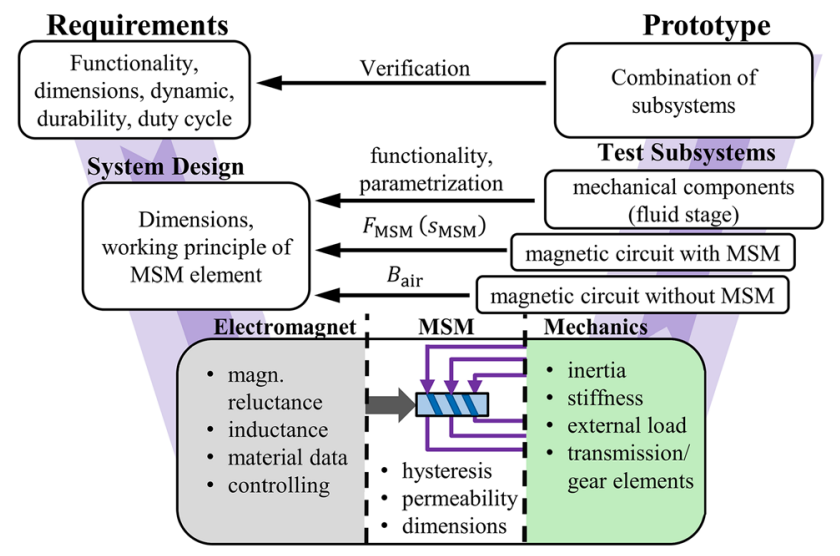

Fig. 1 Illustration of the design process according to the V-model for a valve based on an MSM actuator is important, which is mostly challenging in the design process, because the MSM alloys tend to lose their effect at temperatures between 60 and $80{ }^{\circ} \mathrm{C}$ [7]. Many possible operating principles of MSM alloys [8] result in a large number of valve concepts. In this paper, the working principle that is most promising to meet the requirements on pneumatic valves, namely a magnetic-induced elongation and a mechanical resetting by a spring, is considered. By means of a morphological analysis, a summary of valve concepts was derived and evaluated with respect to the criteria that can be derived from the requirement analysis: available stroke and force of the MSM element, tightness, dynamics and installation size. As the MSM elements cannot be manufactured in arbitrary sizes and shapes, it is advisable to apply an evaluation based on empirical calculations taking into account the limitations in dimension, strain and force of the MSM element. To enable this quantitative evaluation, an initial design of the electromagnet (EM) based on 3D-FEM analysis as depicted in Fig. 2a was created. The concept of the fluid stage that was best rated is shown in Fig. 2b. It includes a lever that enables the required "normally closed" functionality. The sizing process of a pneumatic valve whilst considering the concepts of both the actuator and the valve, implies a description of the EM, the magneto-mechanical behavior of the MSM element and the mechanical load resulting from the fluid stage. In case of a step by step development of the system model, it is possible to identify influencing variables and to analyze causes of deviations between the simulation and experiment. This is fundamentally to understand the interactions between the different domains when simulating the whole system.

\section{MSM Model of the Bivariate Hysteresis}

Besides the hysteresis of the magneto-mechanical behavior, some other characteristics of the MSM alloys are challenging in the design process. Such challenges include, for example, the limitations in shape and dimension, the

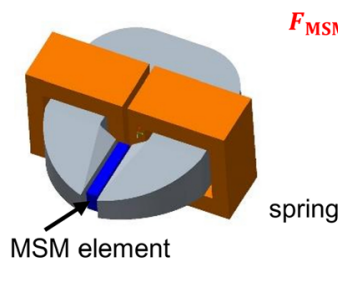

(a)

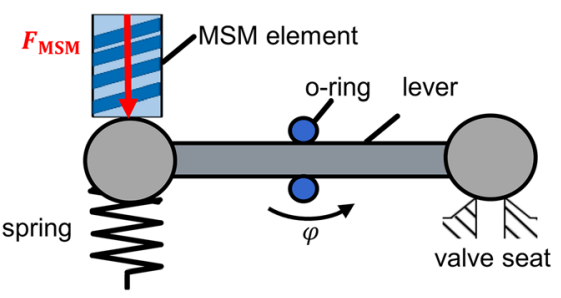

(b)
Fig. 2 Illustration of a the initial actuator design consisting of a c-shaped magnet and two electrically parallel-connected coils and of b the valve concept consisting of an o-ring beared lever. The MSM element in the air gab of the electromagnet will actuate the valve at one side of the lever and will be reset by a spring 
dependency of the work output on temperature as well as the magneto-mechanical characteristic, that diverge between each sample. Therefore, a lumped multi-domain network model which can be easily parametrized is desirable. For this purpose, an MSM model was developed [9], based on a scalar bivariate extension of the Tellinen hysteresis [10].

\section{System Model of the Pneumatic Valve}

Based on the initial design of the valve with the MSM element of the size $2 \times 3 \times 12 \mathrm{~mm}^{3}$ a simulation network model of the whole system, as shown in Fig. 3, including the EM, the MSM model and the fluid stage were built and experimentally analyzed. The EM was described by means of a reluctance network including magnetic inductance elements to take into account eddy current effects. The details of the development, validation and a first optimization approach of the EM are described in [11]. The fluid stage was characterized by a network consisting of basic mechanical elements (inertias, linear stiffness and damping elements, as well as end stops).

\section{Test Rigs for Parametrization and Validation of the System Model}

An empirically sized demonstrator was designed based on rough dimensions to parametrize the lever that is seated in the O-ring. For the parametrization, the test rig shown in Fig. 4 was designed, in which a force sensor can be manually or electrically driven against one of the spheres of the lever. The rotational spring rate $k_{\mathrm{OR}}$ of the O-ring bearing was calculated based on a series of static tests and torque equation considering the influence of the spring while the damping factor $b_{\mathrm{OR}}$ was determined based on a series of dynamic tests at different operational speeds $v_{\text {Fix }}$. Therefore, the value of factor $b_{O R}$ in simulation model was fitted according to the measured curve of $F_{\mathrm{Fix}}\left(x_{\mathrm{Fix}}\right)$.

For the validation of the whole-valve model, the test rig was adapted by replacing the force sensor with the actuator and repositioning the displacement sensor as illustrated in Fig. 5. The actuator was connected to a simple electronics including a MOSFET and a free-wheeling diode to provide a rectangular pulse voltage source with a width of $20 \mathrm{~ms}$ and various amplitudes of $U=[8,10,12,16,20] \mathrm{V}$. The measured voltage at the actuator were used as input for the system model. The measured displacement is comparable with the simulated stroke of the sphere, which is intended to seal the valve.

\section{Functional Prototype of the Valve Including an Optimized Actuator}

By means of the simulation model, a functional prototype of the valve was designed and manufactured as depicted in Fig. 6. The spring of the fluid stage was sized based on a parameter analysis. The design of the actuator was improved by placing the coils close to the MSM element to reduce the stray fluxes. With the reluctance model that was adapted according to the improved actuator design, a multicriterial optimization with the objective function $y$ from (1) was done based on simulation results for a voltage step function from 0 to $48 \mathrm{~V}$.

$\boldsymbol{y}=\left[\begin{array}{c}\int_{t_{0}}^{t_{\text {end }}}\left|B_{\text {air,ref }}-B_{\text {air }, \text { it }}(t)\right| d t, \\ \frac{1}{D}\end{array}\right]$
Fig. 3 Structure of the simulation model in accordance with the highest level of detail of the V-model in Fig. 1. For the sake of a clear view, the magnetic inductance elements are not depicted

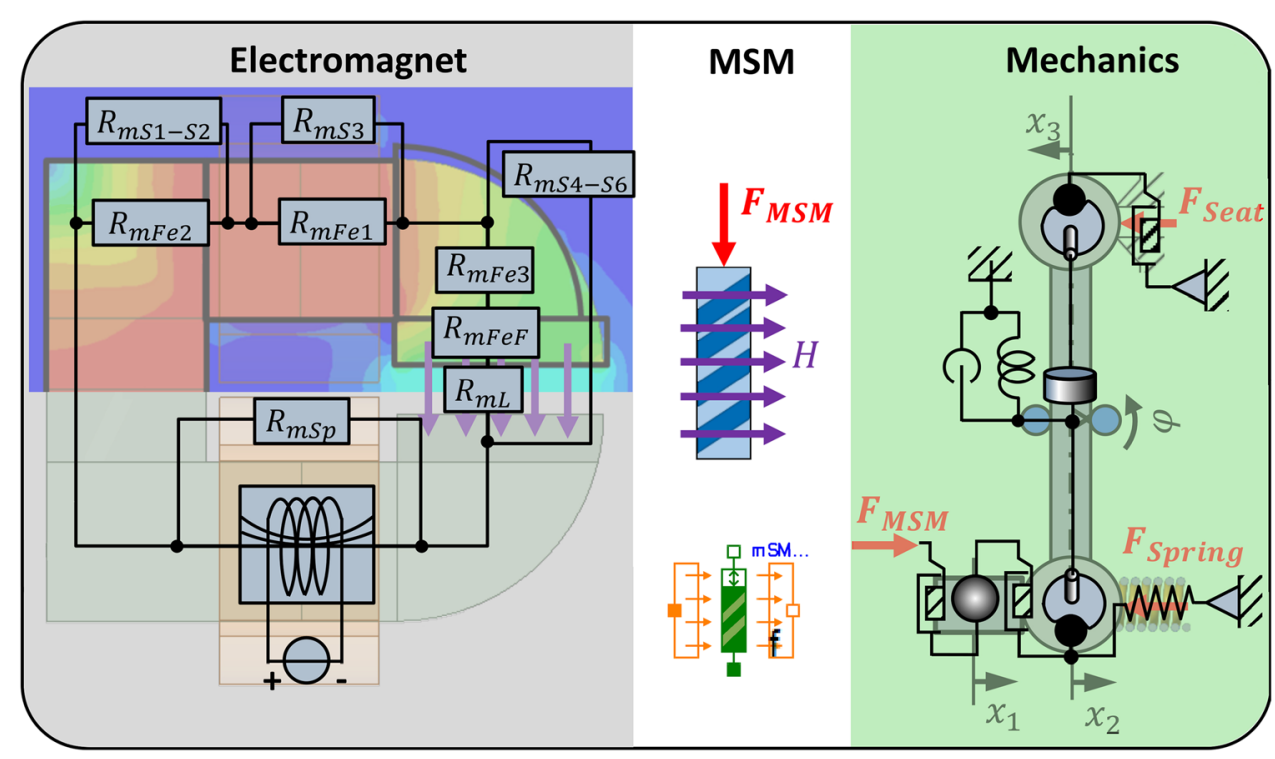


Fig. 4 Schematic illustration of the test rig for parametrization of lever of the fluid stage that is seated in an O-ring. The lever was manually driven against the force sensor to identify the stiffness $k_{O R}$ and electrically driven cossesponding to the displacement profile as shown in the diagram on the right side.

Fig. 5 Illustration of the test rig for validation of the valve by a scheme of the adapted set-up that is shown in Fig. 4 and $\mathbf{b}$ a circuit diagram consisting of a signal generator and a MOSFET to provide a rectangular voltage signal and a shunt for measuring the electrical current

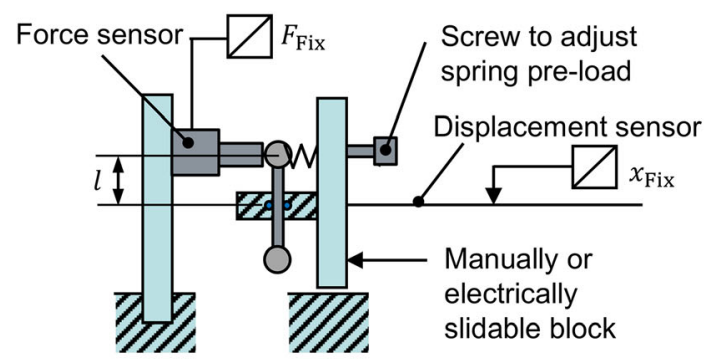

(a)

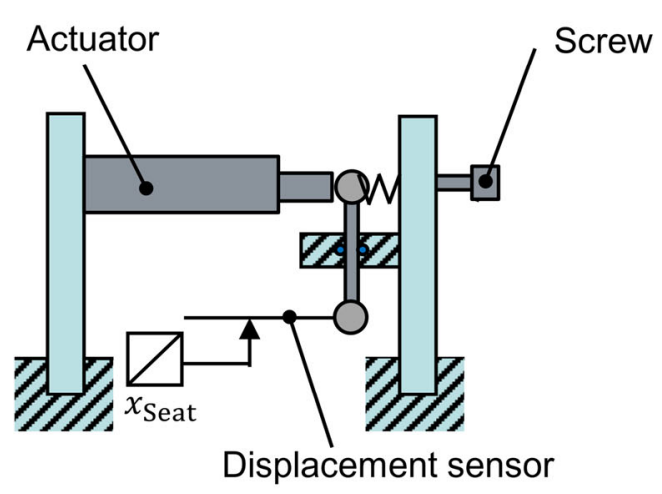

(a)

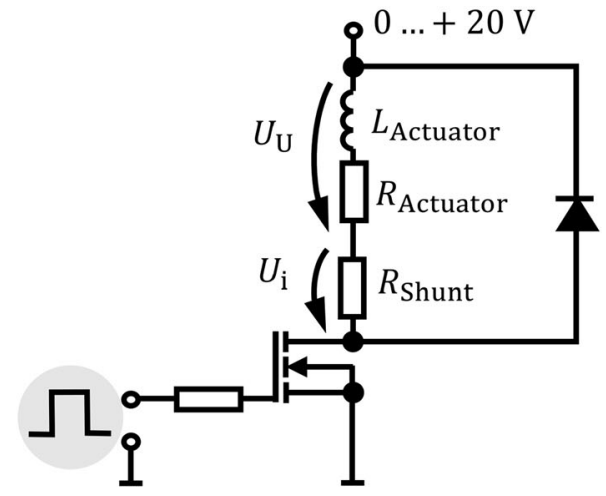

(b)
Motor controlled curve $v_{\mathrm{Fix}}$ and resulting displacement $x_{\text {Fix }}$ for dynamic measurement:

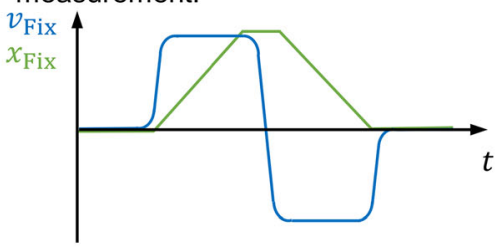

(b)
Fig. 6 Sectional view of the functional prototype of the valve of the $\mathbf{a} C A D$ and $\mathbf{b}$ a drawing of the fluid stage

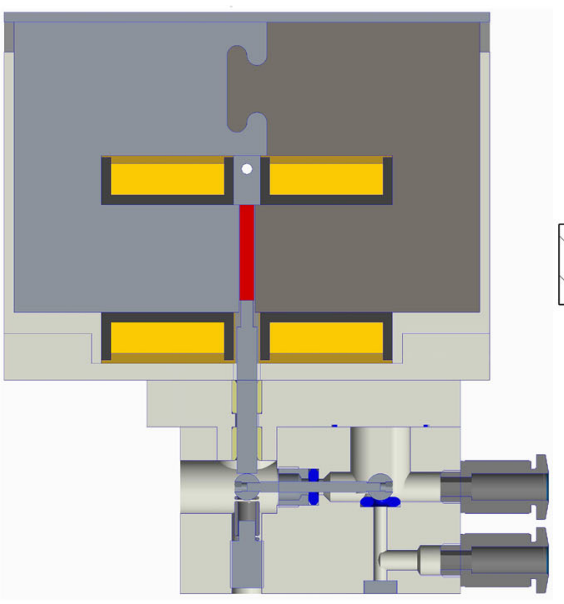

(a)

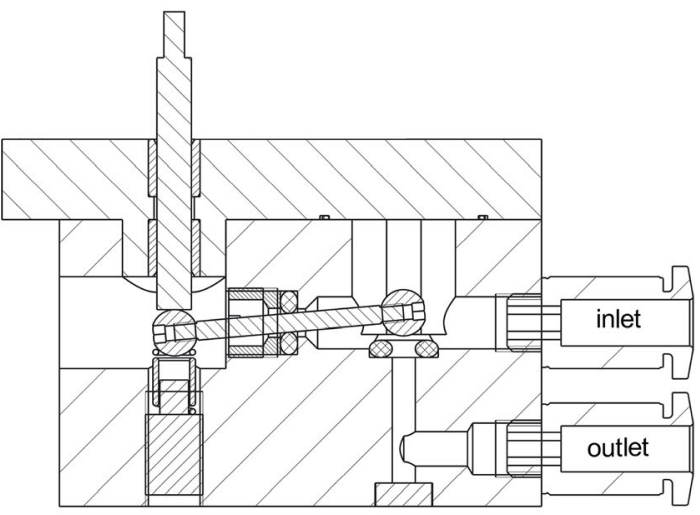

(b)

The formulation of the first objective function $y_{1}$ as the time integral of the absolute deviation between the magnetic flux density of the iteration $B_{\text {air,it }}$ to the reference $B_{\text {air,ref }}$ has the advantage to consider both requirements: the minimization of the response time and providing the necessary magnetic flux density $B_{\text {MSM }}$ for MSM actuation. The value of $B_{\text {MSM }}$ was simulated with the MSM model to be $1 \mathrm{~T}$ at an external mechanical load of $10 \mathrm{~N}$ approximately needed for opening the valve. Assuming the magnetomotive force within the air gap of the EM with and without the MSM element is nearly equal, the required reference $B_{\text {air,ref }}$ can be calculated using the following equation:

$B_{\text {air }, \text { ref }}=\frac{\delta_{\text {air }}\left(A_{\mathrm{MSM}}+A_{\text {air }}\right)-\delta_{\mathrm{MSM}} A_{\mathrm{air}}}{2 \delta_{\mathrm{air}} A_{\mathrm{air}}} B_{\mathrm{MSM}}$,

where $\delta_{\text {air }}$ and $A_{\text {air }}$ are the thickness and the flux-normal area of the air gap respectively $\delta_{\mathrm{MSM}}$ and $A_{\mathrm{MSM}}$ of the MSM element. The second objective function $y_{2}$ includes 
the aims to maximize the duty cycle $D$. The duty cycle $D$ including a reference time of $600 \mathrm{~s}$ according to DIN VDE0580 is defined by:

$D=\frac{t_{\text {on }}}{600 \mathrm{~s}}$.

The time $t_{\mathrm{on}}$ corresponds to the maximal permitted operation time to prevent critical overheating and can be calculated based on the assumption of an intermittent operation with:

$t_{\mathrm{on}}=-\tau_{\mathrm{th}} \times \ln \left[1-\frac{\Delta T_{\mathrm{per}}}{\Delta T_{\mathrm{s}}}\left(1-e^{-\frac{t_{\mathrm{on}}+t_{\mathrm{off}}}{\tau_{\mathrm{th}}}}\right)\right]$

where $\tau_{t h}$ and $\Delta T_{s}$ are the characterizing time constant and the steady-state temperature for the heating, $\Delta T_{\text {per }}$ is the permissible temperature and $t_{\text {off }}$ equals to the reference time of $600 \mathrm{~s}$ reduced by the time $t_{\mathrm{on}}$. For further details consider [12]. Since the MSM element is very close to heat source, i.e. the coils, a permissible temperature of $\Delta T_{\text {per }}=$ $50 \mathrm{~K}$ is considered because the performance of the MSM alloy decreases at higher temperatures.

A functional prototype of the valve system including the optimized actuator was manufactured. The housing of the MSM-based actuator was made by Multi-Jet-Moulding while the fluid stage was produced on a milling machine. The spring is adjustable by a screw while the position of the MSM element with reference to the fluid stage can be shifted by inserting foils between the main body and the cap of the actuator housing. The valve seat consists of an O-ring to compensate deviation of the proper position between the lever and the seat due to manufacturing errors.

\section{Results and Discussion}

\section{Parametrization of the Lever and Validation of the System Model}

The results of the parametrization of the lever are shown in Fig. 7. The average stiffness of the O-Ring bearing is approximately $k_{\mathrm{OR}}=0.6 \mathrm{Nmm} /{ }^{\circ}$ as indicated in Fig. 7a.
The high values of $k_{\mathrm{OR}}$ for small changes in the levers angle $\Delta \varphi$ are caused by the situation that the lever hits the housing for the first settings of the test series. Therefore, the average value was calculated for the range of $2^{\circ}<\Delta \varphi<12^{\circ}$. The results of the damping factors $b_{\mathrm{OR}}$ in Fig. $7 \mathrm{~b}$ show that the damping decreases with a rising speed of operation. The reason for this behavior is the socalled stress relaxation. This is a typical phenomenon for visco-elastic materials the O-Ring is made of and is characterized by a decreasing stress with time while keeping a constant state of deformation. The higher is the operational speed, the less is the occurrence of this effect. Due to the limitation of the test rig, speeds over $v=6 \frac{\mathrm{mm}}{\mathrm{s}}$ could not be tested. A test by free oscillation is not possible because of the very small inertia of the lever. As the damping factor $b_{\mathrm{OR}}$ approaches an end value, the damping factor was assumed to be $b_{\mathrm{OR}}=0.003 \mathrm{Nms} / \mathrm{rad}$.

In Fig. 8 the simulation results of the system model, fitted with the values of $k_{\mathrm{OR}}$ and $b_{\mathrm{OR}}$, are displayed in comparison to the measurement results. The good accordance of the current $i$ leads to the assumption that the dynamic behavior of the magnetic circuit of the simulation confirms to the measurement. But the stroke path exhibits deviations, both static and dynamic. The deviation of the maximal stroke $x_{\text {seat }}$ is about $20 \%$, a time delay in start of expansion and compression can be noticed and in contrast to the measurement the MSM element is not fully reset in the simulation. The primary cause can be explained by the inaccuracy of parametrization of the O-ring bearing. The visco-elastic material of the O-ring bearing behaves frequency- and temperature-dependent and possesses stress relaxation, which is very complex to describe. Furthermore, the actual initial state and position of the MSM are not validated. Thus, it is important to provide appropriate adjustment elements. The deviations in the simulation results can be understood by observing the trajectory of the MSM strain as exemplary illustrated for input pulse signal of $U=20 \mathrm{~V}$ in Fig. 9. In the simulation a time delay of $t_{0}=1 \mathrm{~ms}$ of the voltage source is implemented to reach the initial mechanical steady-state, and the MSM element gets fully compressed and loaded by $\sigma=0.8 \mathrm{MPa}$. As
Fig. 7 Experimental results of $\mathbf{a}$ the stiffness $k_{O R}$ and $\mathbf{b}$ the damping factor $b_{O R}$ against different operational speeds $v$ according to the test rigs in Figs. 3, 4

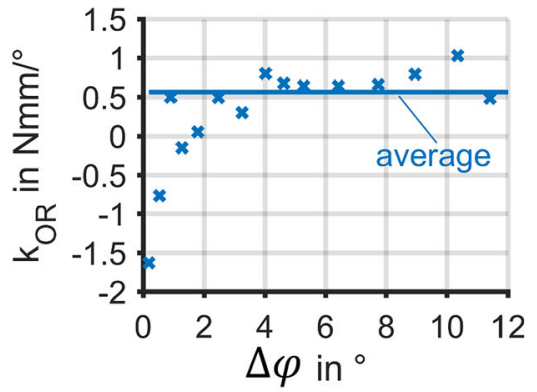

(a)

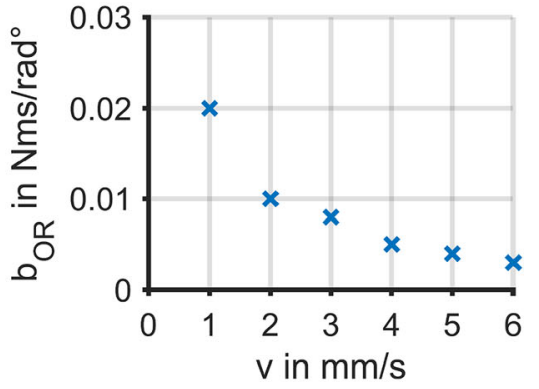

(b) 
Fig. 8 Comparison between the measurements (solid lines) and simulation results (dashed lines) of the test rig including the actuator and the lever demonstrator for a excitation current $i(t)$ for different voltage levels and $\mathbf{b}$ the sphere stroke $x_{\text {seat }}(t)$. The results are colored in gradations from light to dark corresponding to the set value of $U=[8,10,12,16,20] \mathrm{V}$

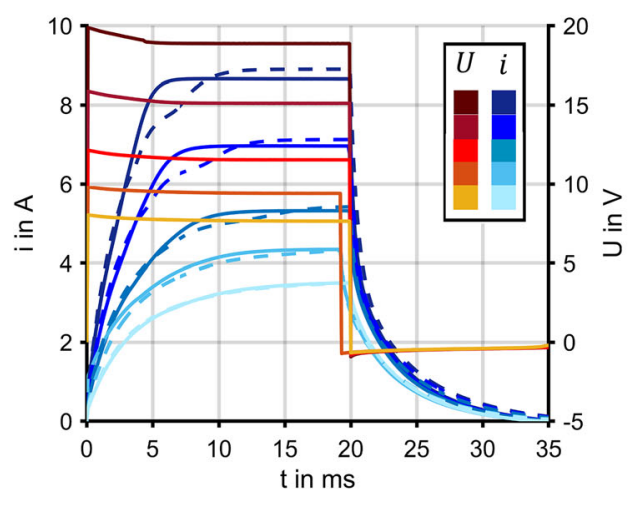

(a)

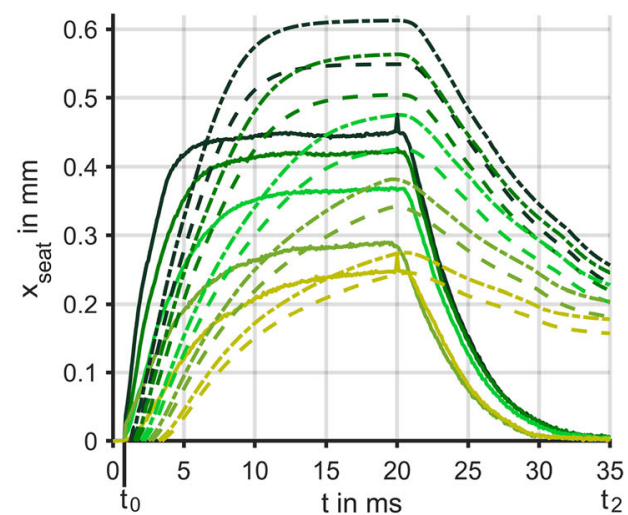

(b)

Fig. 9 Trajectory of MSM strain $\varepsilon=f(B, \sigma)$ for the simulation input pulse signal of $U=20 \mathrm{~V}$ demonstrated against the limiting surfaces. A 2D-plot of the isosurface of $B$ at time $t_{2}$ is shown to clarify that the MSM element is not fully reset at the end of the switching operation

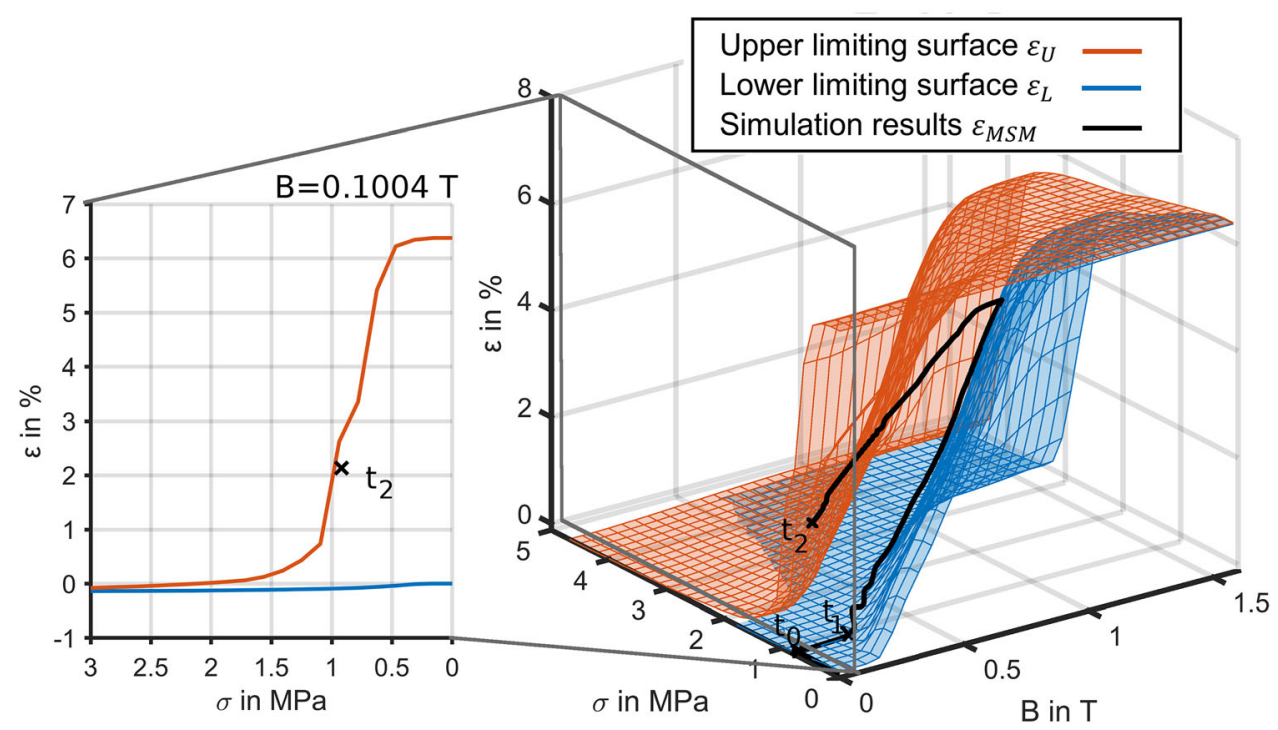

observable by the lower limiting surface, the MSM first starts to elongate after the magnetic flux density $B$ is greater than $0.25 \mathrm{~T}\left(t>t_{1}\right)$. At the end of the actuation cycle $\left(t=t_{2}\right)$, a magnetic field still exists. A high-mechanical load $\sigma$ of $2 \mathrm{MPa}$ would be necessary to reset the MSM element.

\section{Characterization of the Optimized Actuator}

According to Eq. (3) the duty cycle $D$ could be increased by optimization from less than $1 \%$ to $5 \%$. The main influence was to reduce the necessary power supply by improving the magnetic-static characteristics, as the simulation results of the magnet flux density $\Phi_{\text {air }}$ against the magnetomotive force $\Theta$ in Fig. 10a show. Furthermore the response time of the EM could be reduced. For the actuation of the MSM element, a magnetic flux density in the air gap of $0.6 \mathrm{~T}$ is required. With a voltage supply of $48 \mathrm{~V}$ this value is reached after $1.2 \mathrm{~ms}$ with the initial EM design and after $0.7 \mathrm{~ms}$ with the optimized EM design, as shown in Fig. 10b.

\section{Characterization of the Nominal Flow Rate}

A maximum volume flow of $210 \mathrm{Nl} / \mathrm{min}$ at an inlet pressure of $8 \mathrm{bar}_{\mathrm{abs}}$ was reached at a current $i$ of 7.6 A. While the valve is not thermally resistant for continuous operation, the flow rate characterization according to ISO 6358 using the test rig depicted in Fig. 11b is nearly impossible. Nevertheless, the nominal volume flow can be estimated by comparing the measured volume flow of the valve for random pressure rates with the flow curves characterized with the fluid stage for different openings at an inlet pressure of $7 \mathrm{bar}_{\mathrm{abs}}$. Therefore, the opening of the fluid stage was adjusted by fixing the push rod with an iron slab with four screws, as it can be seen in Fig. 11b. The distance and parallelism between the iron slab and the housing of the fluid stage were ensured by gauge blocks. The setting at which no volume flow could be measured was specified as closed position. With respect to the closed position, the push rod was set in the variable distance steps between 0.1 $\mathrm{mm}$ and $1 \mathrm{~mm}$. The results of the characterization of the valve are depicted in Fig. 11c. The random measured 
Fig. 10 Comparison between the initial (solid line) and optimized (dashed line) EM design by a the simulation results of the magneto-static characteristic $\Phi_{\text {air }}(\Theta)$ and $\mathbf{b}$ the measured magneto-dynamic behavior $B(t)$ for a voltage supply of $48 \mathrm{~V}$

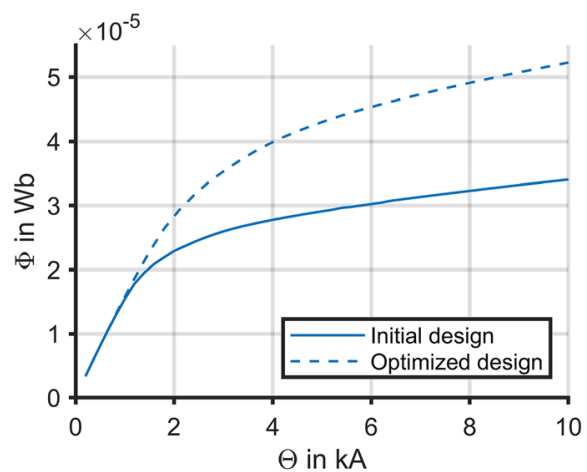

(a)

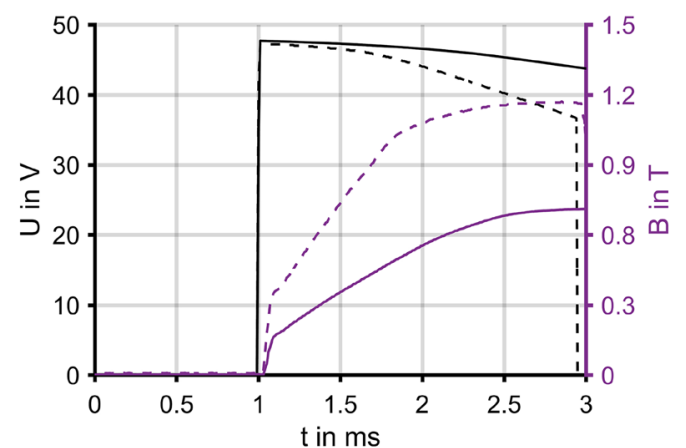

(b)

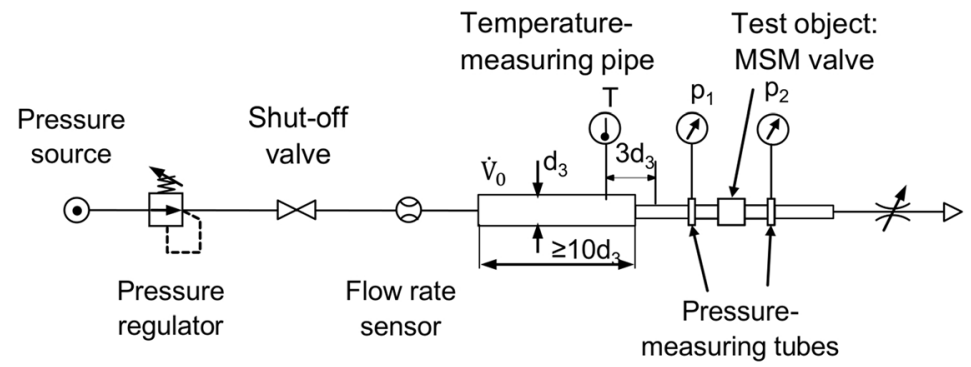

(a)

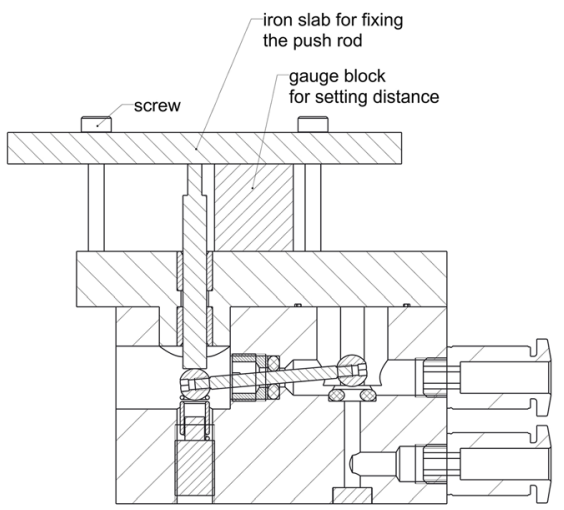

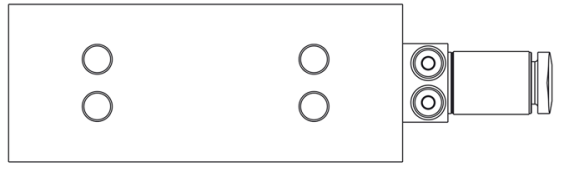

(b)

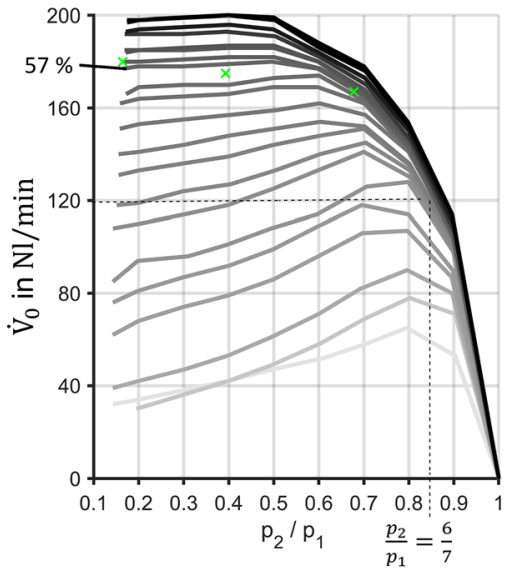

Legend

Fluid stage for different openings MSM-Valve for random pressure rates

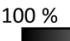

(c) volume flows for the valve including the MSM-based actuator are aligned with the flow curve at the opening of $57 \%$. The nominal volume flow at pressure rate of $p_{2} / p_{1}=$ $6 / 7$ is $120 \mathrm{Nl} / \mathrm{min}$.

\section{Conclusion}

In conclusion, this contribution presents an approach for designing a fast-switching pneumatic valve based on an MSM actuator. A functional prototype of the MSM actuator-based valve is developed by means of a simulation network of the valve system including the electromagnet, MSM model and fluid stage. An optimization of the EM was done to aim for high dynamics and duty cycle. The 
experimental analysis of the functional prototype show, that the flow characteristics of established fast-switching pneumatic valve can be met. It is discussed which challenges can already be met and what further steps are necessary to facilitate the design progress and to enable the maximum benefit of the special characteristics of MSM alloy. The mechanical analysis of a demonstrator of the valve show that small deviations between the simulation model and the experiment have a high impact regarding the strain and dynamics. These uncertainties in combination with the variations in the characteristics of the MSM alloy due to fabrication process hinder a precise sizing of the components. An advancement of the MSM model to respect for aspects such as dependency on temperature, life time and mean variation of the hysteresis according to the sample would enable a more certain sizing of the valve. However, the description of the system by a network model is still applicable for sizing of the valve as required adjustment elements can be identified.

Acknowledgements Open Access funding provided by Projekt DEAL. The authors gratefully acknowledge the funding and support of Bundesministerium für Bildung und Forschung (BMBF) under Contract No. FKZ 03ZZ1010D, FKZ 03ZZ1010F and FKZ 03ZZ1010G.

Open Access This article is licensed under a Creative Commons Attribution 4.0 International License, which permits use, sharing, adaptation, distribution and reproduction in any medium or format, as long as you give appropriate credit to the original author(s) and the source, provide a link to the Creative Commons licence, and indicate if changes were made. The images or other third party material in this article are included in the article's Creative Commons licence, unless indicated otherwise in a credit line to the material. If material is not included in the article's Creative Commons licence and your intended use is not permitted by statutory regulation or exceeds the permitted use, you will need to obtain permission directly from the copyright holder. To view a copy of this licence, visit http://creativecommons. org/licenses/by/4.0/.

\section{References}

1. Schiepp T, Pagounis E, Laufenberg M (2014) Magnetic shape memory actuators for fluidic applications; 9th international fluid power conference, Aachen, Germany. https://www.etogruppe.
com/images/MAGNETOSHAPE_Ver\%C3\%B6ffentlichungen/ Magnetic_Shape_Memory_Actuators_for_Fluidic_Applications. pdf. Accessed 13 Feb 2020

2. Breisch S, Tueysuez A, Schautzgy M, Laufenberg M (2018) Combined Thermal and Magnetic Tripping of MSM. Actuator 2018; 16th International Conference on new actuators, Bremen, Germany, pp 1-4. https://ieeexplore.ieee.org/stamp/stamp. jsp? $\mathrm{tp}=\&$ arnumber $=8470802 \&$ isnumber $=8470458$. Accessed 3 Dec 2019

3. Ullakko K, Wendell L, Smith A, Müllner P, Hampikian G (2012) A Magnetic shape memory micropump: contact-free, and compatible with PCR and human DNA profiling. Smart Mater Struct 21:115020. https://doi.org/10.1088/0964-1726/21/11/115020

4. Holz B, Riccardi L, Janocha H, Naso D (2012) MSM actuators: design rules and control strategies. Adv Eng Mater 14:668-681. https://doi.org/10.1002/adem.201200045

5. Kiefer B, Lagoudas D (2009) Modeling the coupled strain and magnetization response of magnetic shape memory alloys under magnetomechanical loading. J Intell Mater Syst Struct 20:143-170. https://doi.org/10.1177/1045389X07086688

6. Couch RN, Chopra I (2007) A quasi-static model for NiMnGa magnetic shape memory alloy. Smart Mater Struct 16(1):S11S21. https://doi.org/10.1088/0964-1726/16/1/S02

7. Pagounis E, Chulist R, Szerba MJ, Laufenberg M (2014) Hightemperature magnetic shape memory actuation in a Ni-Mn-Ga single crystal. Scripta Mater 83:29-32. https://doi.org/10.1016/j. jallcom.2015.02.090

8. Schlüter K, Holz B, Raatz A (2012) Principle design of actuators driven by magnetic shape memory alloys. Adv Eng Mater 14:682-686. https://doi.org/10.1002/adem.201200078

9. Effner A, Zawada T, Ehle F, Neubert H, Weber J (2018) Entwicklung und Anwendung einer SimulationX-Bibliothek für Magnetformgedächtnislegierungen (Development and Applocation of a SimulationX library for magnetic shape memory alloys) ESI SimulationX Conference, p 377. https://www.simulationx.de/ esi-foren/esi-simulationx-conference/2018.html. Accessed 3 Dec 2019

10. Ziske J, Ehle F, Neubert H, Price A, Lienig J (2015) A simple phenomenological model for magnetic shape memory actuators. IEEE Trans Magn 51(1):1-8. https://doi.org/10.1109/TMAG. 2014.2338833

11. Effner A, Tautenhahn R, Weber J, Schiepp T, Schnetzler R, Laufenberg M (2018) Fast Switching pneumatic valves driven by magnetic shape memory materials. 11. Internationales fluidtechnisches kolloquium aachen. https://publications.rwth-aachen.de/ record/724070/files/724070.pdf. Accessed 3 Dec 2019

12. Kallenbach E, Eick R, Quendt P, Ströhla T, Feindt K (2012) Elektromagnete (Electromagnets), vol 4. Springer, Heidelberg

Publisher's Note Springer Nature remains neutral with regard to jurisdictional claims in published maps and institutional affiliations. 